\title{
Public Health/ Health Information Exchange Collaborative: A Model for Advancing Public Health Practice
}

\section{Charles Magruder, Centers for Disease Control and Prevention (CDC)}

In early 2007, senior leadership in CDC's new Office of Surveillance, Epidemiology and Laboratory Services (OSELS) examined ways to improve biosurveillance capabilities in collaboration with public and private partners. After careful consideration, it was determined that many key aspects of surveillance could be enhanced through Health Information Exchange (HIE) interactions. Approximately one year later in February 2008, CDC awarded three 1-year contracts (with options for 4 additional years) to address this area through an initial focus on current public health priorities, such as pandemic influenza. Although only three sites were selected to become Public Health/HIE Collaboratives, the high number of qualified applicants indicated tremendous potential for future growth.

The initial awardees were diverse in many ways. For example, one collaborative proposed development of an HIE system in a community of about 500,000 people in Washington State, while another focused its efforts on a large, metropolitan area in Indiana. The third collaborative proposed development of a statewide HIE in New York that would include several communities of varying sizes. In addition, the lead partner for each is different-representing the private sector, a not-for-profit research institute, and a state health department, respectively.

From its inception, OSELS has worked collaboratively with various programs at Health and Human Services (HHS), including the Office of the National Coordinator for Health Information Technology (ONC). In addition, OSELS has been committed to promoting the appropriate use of Health IT standards and providing the support needed to launch critical demonstration projects. At the same time, OSELS is focusing on issues that are unique to CDC and working with the Biosurveillance Coordination Unit and other internal CDC departments. Further, OSELS coordinates its activities with state and local health authorities in the areas supported by the Public Health/HIE Collaborative and encourages other partners, such as the Association of State and Territorial Health Officials, the Council of State and Territorial Epidemiologists, and the National Association of City and County Health Officials, to participate in these efforts.

In a relatively short time, the collaborative have made great strides in supporting a variety of HHS and CDC initiatives and objectives. For example, an assessment of Minimum Biosurveillance Data Set acquisition capability has been completed, and plans to address shortcomings have been developed. In addition, an implementation guide for managing and transporting these data was created in accordance with mandated Health Information Technology Standards Panel (HITSP). Some unanticipated deliverables have been produced through this process as well. For example, the Regenstrief Institute, the coordinator of the Indiana Public Health/HIE collaborative, demonstrated a natural language processor for electronic laboratory reports, a breakthrough that could reduce costs and increase the amount of useable data received. 
In addition, OSELS has strengthened its collaborative efforts with other federal agencies, working closely with HHS's Office of Science and Data Policy to develop a more comprehensive evaluation process. OSELS also continues to make progress in demonstrating various aspects of the biosurveillance use case, showcasing advances in public health practice that are possible with HIE collaboration. In December 2008, OSELS successfully demonstrated its capabilities in this area at an HHS Nationwide Health Information Network conference in Washington, DC. A few months later at a Healthcare Information and Management Systems Society meeting, OSELS demonstrated its ability to use appropriate standards to transmit deidentified, aggregated data from individual health care sources through the HIE to state and local health departments. Once the data were analyzed at the local and state levels, these were sent to CDC for further analysis from a national perspective. Subsequently, CDC used the resulting national trend data to quickly send out public health alerts to appropriate areas of the country. Thus, OSELS demonstrated a functional, bidirectional communication process; one in which population data can be assessed quickly and important information can be released quickly to public health and health care practitioners.

In August 2009, OSELS completed another demonstration at the PHIN Conference Interoperability Showcase. In this scenario, OSELS emphasized new capabilities that were developed to support H1N1 Influenza surveillance activities, including a new, standardized format for H1N1 surveillance called the Geocoded Interoperable Population Summary Exchange (GIPSE). In September 2009, the Public Health/HIE Collaborative began using the GIPSE format to send H1N1 Influenza data to CDC. Currently, the members of the collaborative are working together to modify the GIPSE format to address new H1N1 Influenza challenges identified by CDC and epidemiologists working in state and local health departments. Foremost among these challenges is the need to develop a severity index. At the same time, each member is working on new initiatives that can be supported by HIE collaborations, including enhancements in case reporting, development of a quality use case to address chronic diseases, enhanced alerting capabilities, and development of new biosurveillance formats for other infectious diseases.

Even at this early point in the process, there is sound evidence to substantiate this investment. OSELS will continue to work to improve its preparedness capabilities at local, state, and federal levels and to strengthen its basic infrastructure to support other medical and public health activities.

Charles Magruder, MD, MPH

Medical Epidemiologist

Center for Disease Control (CDC)

National Center for Public Health Informatics

1600 Clifton Road

MS E-68

Atlanta, GA

USA 30047

Email: zgu4@cdc.gov 